\title{
Theoretical improvements for luminosity monitoring at low energies
}

Janusz Gluza*, Michał Gunia

Institute of Physics, University of Silesia, 40-007 Katowice, Poland

E-mail: janusz.gluza@us.edu.pl

\section{Tord Riemann}

Deutsches Elektronen-Synchrotron, DESY, Platanenallee 6, 15738 Zeuthen, Germany

\section{Małgorzata Worek}

Fachbereich C Physik, Bergische Universität Wuppertal,

Gaussstr. 20, D-42097 Wuppertal, Germany

A comparison of theoretical results on NNLO leptonic and hadronic corrections to Bhabha scattering with the Monte Carlo generator BabaYaga@NLO used at meson factories is given. Complete NLO virtual corrections to the $e^{+} e^{-} \rightarrow \mu^{+} \mu^{-} \gamma$ process are discussed.

10th International Symposium on Radiative Corrections (Applications of Quantum Field Theory to Phenomenology) - Radcor2011

September 26-30, 2011

Mamallapuram, India

${ }^{*}$ Speaker. 


\section{Introduction}

This Radcor symposium is devoted mainly to the high energy, LHC physics. However, low energy physics remains an active area of research. There are a few on-going low energy $e^{+} e^{-}$ experiments like the $\Phi$ factory DA $\Phi N E$ (with the KLOE detector) the $B$ factories PEP-II and KEK (with the BaBar detector at SLAC for which the accumulated data are still analysed and the Belle detector at KEK), the charm $/ \tau$ factory BEPC II (detector BES III). For them, to operate properly, a precise calculation of higher order corrections $\sigma_{\text {theory }}$ for the process of Bhabha scattering $\left(e^{+} e^{-} \rightarrow\right.$ $\left.e^{+} e^{-}\right)$is necessary. Then the collider's luminosity $\int L_{t o t} d t=\frac{N_{e x p}}{\sigma_{\text {theory }}}$ can be determined with high accuracy. Here $N_{\exp }$ stands for a number of detected events, $\sigma$ is the theoretical cross section of the chosen reference process. If we know the luminosity accurately in a low energy region, then also the low energy hadron cross sections in $e^{+} e^{-}$annihilation processes $\sigma_{\text {had }}=\frac{N_{\text {had }}}{L_{\text {tot }}}=\sigma_{\text {theory }} \frac{N_{\text {had }}}{N_{\text {exp }}}$ can be inspected ( $N_{\text {had }}$ is the number of measured hadronic events). What follows is that $\sigma_{\text {theory }}$ serves as the calibration parameter in measurements. ${ }^{1}$ Two loop virtual $\mathrm{QED}^{2}$ corrections to the Bhabha scattering are already known [3-16], however, so far their effects have not been applied to realistic physical situations (theoretical groups made comparisons only among virtual corrections, switching off the soft-photon cut-off parameter). For unresolved photons, fermion pairs, or hadrons at NNLO (real emission),

$$
e^{+} e^{-} \rightarrow e^{+} e^{-}(\gamma, \gamma \gamma), \quad e^{+} e^{-}\left(e^{+} e^{-}\right), \quad e^{+} e^{-}\left(f^{+} f^{-}\right), \quad e^{+} e^{-} \text {(hadrons) },
$$

we use the Fortran packages HELAC-PHEGAS (fermion pairs) [17-20], the MC event generator EKHARA [21 - 24] (pion pairs), and the Fortran program BHAGEN-1PH-VAC (photons emission, M.Gunia, H.Czyż, unpublished, based on the generator BHAGEN-1PH [25]). The complete results are compared with the BabaYaga [26-28] MC generator in its recent version BABAYAGA@ NLO [29].

On the other hand, the muon pair production with real photon emission $e^{+} e^{-} \rightarrow \mu^{+} \mu^{-} \gamma$ is an important background and normalization reaction in the measurement of the pion form-factor: $R_{e x p}=\frac{\sigma\left(e^{+} e^{-} \rightarrow \pi^{+} \pi^{-} \gamma\right)}{\sigma\left(e^{+} e^{-} \rightarrow \mu^{+} \mu^{-} \gamma\right)}$. We report on a progress in the determination of complete virtual corrections.

\section{NNLO Bhabha scattering at low energies}

In [30] it has been shown that for assumed realistic event selections the total NNLO massive corrections are relevant for precision luminosity measurements with $10^{-3}$ accuracy. Second, the maximum observed difference between BABAYAGA @ NLO and exact massive NNLO corrections is at the level of $0.07 \%$. When cuts are varied the sum of the missing pieces can reach $0.1 \%$, but for very tight acollinearity cuts only. The electron pair contribution is the largely dominant part of the correction. The muon pair and hadronic corrections are the next-to-important effects and quantitatively on the same grounds. The tau pair contribution is negligible for the energies of meson factories. Since the work [30] has already been reported at two conferences by M. Gunia ("Matter To The Deepest", Ustroń 2011, [31]) and G. Montagna ("PHIPSI11", Novosibirsk 2011,

\footnotetext{
${ }^{1}$ For more details on possible luminosity options at low energies, see the Introduction in [1].

${ }^{2}$ Recently the dominant logarithmic 2-loop electroweak corrections, important at ILC energies, have been computed in [2].
} 
[32]), we focus here on a part of results which has not been discussed there: main aspects of hadronic contributions and narrow resonances, for a complete discussion, see [30].

Specific problems are caused by narrow resonances like $J / \psi, \psi(2 S), \ldots$. Typically, they are very narrow (at the $\mathrm{keV}$ level) and beam spread effetcs (at the $\mathrm{MeV}$ level) may cause that their final effects are diffused. This problem should be study. Here we show that their effects at fixed center of mass energy is dominating.

Narrow resonances with mass $M_{r e s}$ and partial width $\Gamma_{r e s}^{e^{+} e^{-}}$can be described approximately by the ansatz

$$
R_{\text {res }}(z)=\frac{9 \pi}{\alpha^{2}} M_{\text {res }} \Gamma_{r e s}^{e^{+} e^{-}} \delta\left(z-M_{r e s}^{2}\right) .
$$

Based on this, their contributions to the NNLO Bhabha process can be derived from the general formulae of [15]. We discuss here as an example the contribution from the "rest" (genuine irreducible 2-loop corrections, as defined in [15]). According to Eq. (87) of [15] it reads:

$$
\frac{d \sigma_{\mathrm{rest}}}{d \Omega}=\frac{9 \alpha^{2}}{\pi s} \frac{\Gamma_{\mathrm{res}}^{e^{+}} e^{-}}{M_{\mathrm{res}}}\left\{\frac{F_{1}\left(M_{\mathrm{res}}^{2}\right)}{t-M_{\mathrm{res}}^{2}}+\frac{1}{s-M_{\mathrm{res}}^{2}}\left[F_{2}\left(M_{\mathrm{res}}^{2}\right)+F_{3}\left(M_{\mathrm{res}}^{2}\right) \ln \left|1-\frac{M_{\mathrm{res}}^{2}}{s}\right|\right]\right\} .
$$

Let us note that Eq. 2.2 becomes invalid when the center of mass energy comes too close to the position of a resonance, i.e. if $\left(s-M_{\text {res }}^{2}\right) \lesssim \Gamma_{\text {res }}^{e^{+} e^{-}} M_{\text {res. }}$. In the numerical examples, Table 1 , this is not the case.

Table 1: Soft+virtual NNLO contributions $\sigma_{\text {rest,res }}^{\mathrm{NNO}}$ from narrow resonances (n.r.) defined by Eq. (2.2) for the Bhabha process with $\omega / E_{\text {beam }}=10^{-4}$ (in $\mathrm{nb}$ ). The narrow resonance located closest to the center of mass energy of the given collider is included (first column, res) and excluded (second column, res'). The

\begin{tabular}{|c|c|c|c|c|}
\hline & $\sqrt{s}$ & $\sigma_{\text {rest,res }}^{\mathrm{NNLO}}$ & $\sigma_{\text {rest.res }}^{\mathrm{NNLO}}$ & $\overline{\sigma_{B}}$ \\
\hline \multirow{2}{*}{\multicolumn{2}{|c|}{ KLOE 1.020}} & [all n.r.] & {$[$ [n.r. without $\mathrm{J} / \psi(1 \mathrm{~S})]$} & \\
\hline & & -0.04538 & -0.0096 & 529.5 \\
\hline \multirow[t]{2}{*}{ BES } & 3.097 & [all n.r.] & [n.r. without $\mathrm{J} / \psi(1 \mathrm{~S})]$ & \\
\hline & & 228.08 & -0.0258 & 14.75 \\
\hline \multirow[t]{2}{*}{ BES } & 3.650 & [all n.r.] & [n.r. without $\psi(2 \mathrm{~S})]$ & \\
\hline & & -0.1907 & -0.023668 & 123.94 \\
\hline \multirow[t]{2}{*}{ BES } & 3.686 & [all n.r.] & [n.r. without $\psi(2 \mathrm{~S})]$ & \\
\hline & & -62.537 & -0.0254 & 121.53 \\
\hline \multirow[t]{2}{*}{ BaBar } & 10.56 & [all n.r.] & [n.r. without $\Upsilon(4 S)]$ & \\
\hline & & -0.0163 & -0.01438 & 6.744 \\
\hline \multirow[t]{2}{*}{ Belle } & 10.58 & [all n.r.] & [n.r. without $\Upsilon(4 S)]$ & \\
\hline & & 0.04393 & -0.0137 & 6.331 \\
\hline
\end{tabular}
third column contains the Born cross section $\sigma_{B}$.

In Table 1 we show numerical results based on Eq. (2.2). We can see that the contributions from narrow resonances dominate the NNLO Bhabha correction for BES running at $J / \psi$ and $\psi(2 S)$ energies. For the remaining cases narrow resonances contribute below the per mille level when compared to the Born cross section $\sigma_{B}$. For those cases results can be found in [30]. 
We conclude that for experiments performed on top of a narrow resonance, this resonance cannot be treated as a mere correction and more detailed studies have to be performed. These should include the examination of finite width effects, beam spread effects, the estimation of NNNLO corrections, and the accuracy of the vacuum polarisation insertions in a close vicinity of these resonances.

\section{3. $e^{+} e^{-} \rightarrow \mu^{+} \mu^{-} \gamma$ at low energies}

The $e^{+} e^{-} \rightarrow \mu^{+} \mu^{-} \gamma$ process is an ideal benchmark process to test for massive tensor reductions at the 1-loop level. It has (i) two different masses (and thus large difference of scales, up to 7 orders in magnitude); (ii) quasi-collinear regions (due to small electron mass); (iii) small number of diagrams.

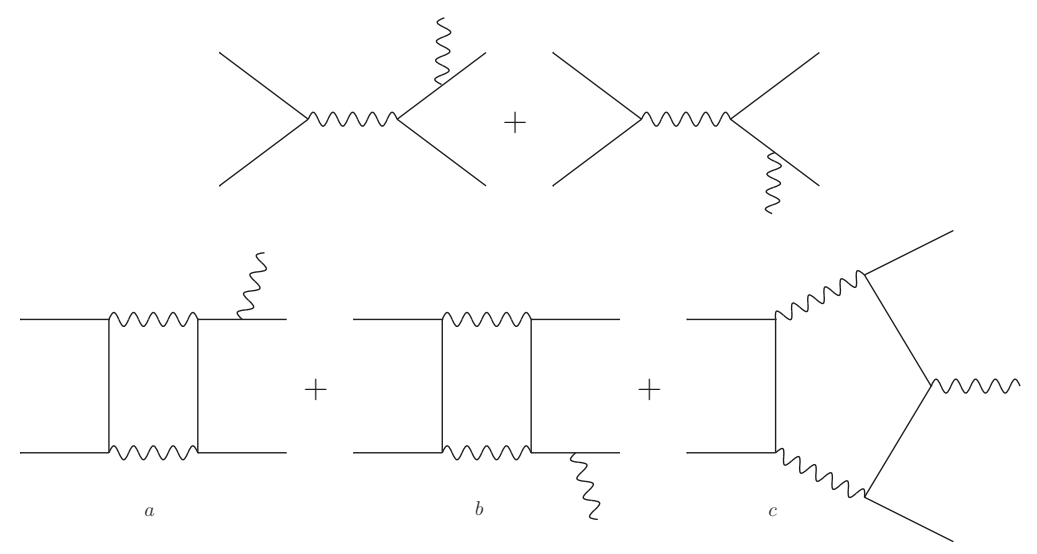

Figure 1: A set of diagrams needed for final state radiation (FSR) gauge invariance of interferences between tree diagrams (upper picture) and four and five point one-loop integrals (below). Here the diagrams are limited to FSR cases, the same property holds if initial state radiation (ISR) amplitudes are present.

There are many tools which give a possibility to obtain automatically cross sections at oneloop level, however, there is still ongoing progress as new demands on precision of calculations require a more and more precise numerical analysis. Here we give an example of such a progress in the calculation of effects including 5-point functions. In Fig.1 a class of diagrams is shown needed for building gauge invariant amplitudes. Let us define the relative accuracy

$$
A=\max \frac{\sum_{i=a, b, c} \Re\left(M_{\text {loop }}^{i} M_{\text {tree }}^{\dagger}\right)}{\min \Re\left(M_{\text {loop }}^{i} M_{\text {tree }}^{\dagger}\right)}
$$

where $i$ stands for (a),(b),(c) in Fig.1. For such a test parameter, with the package LoopTools /FF $[33,34], 2.5 \times 10^{6}$ events have been generated. For Fortran double precision, an accuracy at the level $A=10^{-2}$ has been obtained, and for quadruple precision (with an Intel Fortran compiler), it is $A=10^{-12}$. It is clear that using quadruple precision, a reasonable level of accuracy has been obtained. In Fig. 2 a distribution of muon pairs in $e^{+} e^{-} \rightarrow \mu^{+} \mu^{-} \gamma$ is shown at KLOE kinematics. ${ }^{3}$ Parameters as defined in Table 2 have been used. The results in Figs. 2 and 4 estimate only the

\footnotetext{
${ }^{3}$ Similar plots for the same number of generated events but different distributions can be found in [35]).
} 


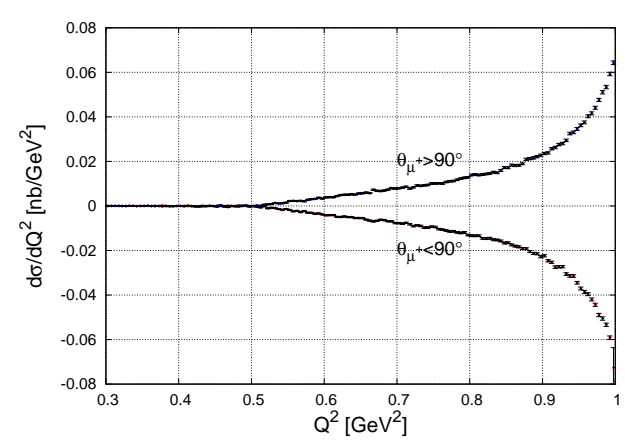

Figure 2: Muon pair distributions including 5-point functions at KLOE. $2.5 \times 10^{6}$ events have been generated. Looptools and FF packages have been used.

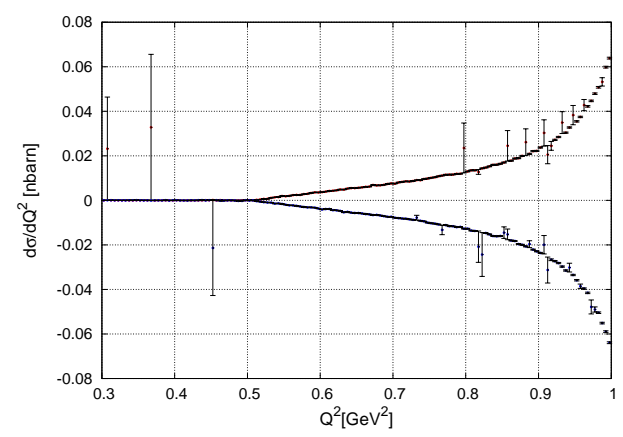

Figure 3: Muon pair distributions including 5-point functions at KLOE. $10^{9}$ events have been generated. Looptools and FF packages have been used.

virtual contributions. However, with an increasing number of generated events some instabilities appear, see Fig.3. Since version 2.2, LoopTools allows to choose in which decomposition five point integrals should be calculated. Implementations of both the Passarino-Veltman [36] and the Denner/Dittmaier [37, 38] schemes exist. The instability problems are due to the appearance of inverse Gram determinants, and their solution is achieved with the alternative reduction scheme [39-41] and its implementation in the package P JFry. ${ }^{4}$

\begin{tabular}{|c|c|c|c|c|}
\hline$E_{C M S}$ & $E_{\gamma, \min }$ & $\theta_{\gamma}$ & $Q^{2}$ & $\theta_{\mu^{ \pm}}$ \\
\hline $1.02 \mathrm{GeV}$ & $0.02 \mathrm{GeV}$ & $0^{\circ}-15^{\circ}, 165^{\circ}-180^{\circ}$ & $0.25-1.06 \mathrm{GeV}^{2}$ & $50^{\circ}-130^{\circ}$ \\
\hline
\end{tabular}

Table 2: Phase-space cuts for KLOE settings used in calculations. $Q^{2}$ is the invariant mass squared of the muon pair.

Using these new reductions, in Fig.4 the same stability of distributions as in Figs.2,3 is obtained. The results are completely stable and well controlled. E.g. the leading inverse Gram determinants $\left|G^{(5)}\right|$ are eliminated in the reduction and small inverse Gram determinants $\left|G^{(4)}\right|$ are avoided using asymptotic expansion.

\footnotetext{
${ }^{4}$ For the evaluation of the scalar integrals we use QCDloop/FF [42]. For further references and some basic comparisons see [43, 34].
} 


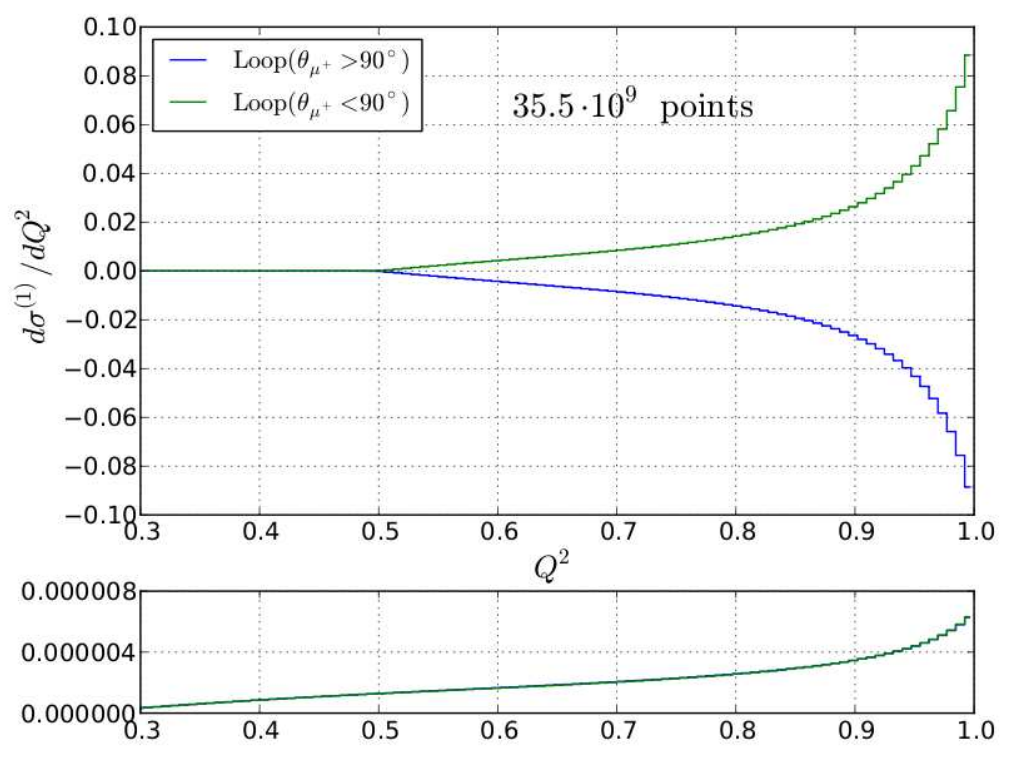

Figure 4: Muon pair distributions including 5-point functions at KLOE. Bottom: absolute error estimate. Approximately $4 \cdot 10^{10}$ events has been generated. PJFry package has been used. Taken from [44].

\section{Summary}

Low energy physics is an important field of activity. Let us mention the measurement of $(g-2)_{\mu}$ and its theoretical calculation, ${ }^{5}$ but also low energy hadron physics, e.g. the determination of form factors. In this context, an important quantity is the pion form factor, see e.g. [45]. In order to describe it properly, experimental data are needed, and for that the process $e^{+} e^{-} \rightarrow \mu^{+} \mu^{-} \gamma$ serves as normalization reaction. We have shown some details on progress in the calculation of this process at the NLO level, including 5-point diagrams. The progress has been possible due to a stable treatment of tensor reductions. Similarly, theoretical progress in calculations of NNLO effects in Bhabha scattering convenience us that the existing generator BabaYaga@NLO is a reliable tool used for luminosity determination at meson factories. Here we have signaled only that some additional work on the understanding of narrow resonance contributions to precise Bhabha scattering studies would be welcome.

\section{Acknowledgments}

JG and TR would like to thank Organizers of the Radcor symposium: D. Indumathi, Prakash Mathews, Andreas Nyffeler and V. Ravindran for their warm hospitality and perfectly prepared conference. We would like to thank: C.C. Calame, G. Montagna, O. Nicrosini, F. Piccinini (the BabaYaga group) and H. Czyż, J. Fleischer, V. Yundin for nice collaborations. This work originated from the activity of the "Working Group on Radiative Corrections and Monte Carlo Generators for Low Energies" [http://www.lnf.infn.it/wg/sighad/]. MW was supported by the Initiative and Networking Fund of the Helmholtz Association, contract HA-101 ("Physics at the Terascale"). This

\footnotetext{
${ }^{5}$ See the contribution by R. Szafron, these proceedings.
} 
work is also supported in part by Sonderforschungsbereich/Transregio SFB/TRR 9 of DFG "Computergestützte Theoretische Teilchenphysik", European Initial Training Network LHCPHENOnet PITN-GA-2010-264564 and by Polish Ministry of Science and Higher Education from budget for science for 2010-2013 under grant number N N202 102638.

\section{References}

[1] S. Actis et al. (Working Group on Radiative Corrections and Monte Carlo Generators for Low Energies), Eur.Phys.J. C66, 585 (2010), arXiv: 0912.0749

[2] A. Penin, G. Ryan, JHEP 1111, 081 (2011), 1112.2171

[3] E.W.N. Glover, J.B. Tausk, J.J. Van der Bij, Phys. Lett. B516, 33 (2001), hep-ph / 0106052

[4] R. Bonciani, A. Ferroglia, P. Mastrolia, E. Remiddi, J. van der Bij, Nucl. Phys. B681, 261 (2004), hep-ph/0310333

[5] M. Czakon, J. Gluza, T. Riemann, Phys. Rev. D71, 073009 (2005), hep-ph / 0412164

[6] A.A. Penin, Phys. Rev. Lett. 95, 010408 (2005), hep-ph / 0501120

[7] R. Bonciani, A. Ferroglia, P. Mastrolia, E. Remiddi, J. van der Bij, Nucl. Phys. B716, 280 (2005), hep-ph/0411321v2

[8] R. Bonciani, A. Ferroglia, P. Mastrolia, E. Remiddi, J. van der Bij, Nucl. Phys. B701, 121 (2004), hep-ph/0405275

[9] M. Czakon, J. Gluza, T. Riemann, Nucl. Phys. B751, 1 (2006), hep-ph/ 0604101

[10] S. Actis, M. Czakon, J. Gluza, T. Riemann, Nucl. Phys. B786, 26 (2007), 0704.240 0v2

[11] T. Becher, K. Melnikov, JHEP 06, 084 (2007), arXiv: 0704.3582 [hep-ph ]

[12] R. Bonciani, A. Ferroglia, A.A. Penin, Phys. Rev. Lett. 100, 131601 (2008), arXiv : 0710.4775

[13] S. Actis, M. Czakon, J. Gluza, T. Riemann, Acta Phys. Polon. B38, 3517 (2007), arXiv:0710.5111 [hep-ph]

[14] S. Actis, M. Czakon, J. Gluza, T. Riemann, Phys. Rev. Lett. 100, 131602 (2008), 0711.3847

[15] S. Actis, M. Czakon, J. Gluza, T. Riemann, Phys. Rev. D78, 085019 (2008), 0807 . 4691

[16] J.H. Kühn, S. Uccirati, Nucl. Phys. B806, 300 (2009), arXiv: 0807.1284

[17] A. Kanaki, C.G. Papadopoulos, Comput. Phys. Commun. 132, 306 (2000), hep-ph / 0002082

[18] C.G. Papadopoulos, Comput. Phys. Commun. 137, 247 (2001), hep-ph/ 0007335

[19] C.G. Papadopoulos, M. Worek, Eur. Phys. J. C50, 843 (2007), hep-ph/ 0512150

[20] A. Cafarella, C.G. Papadopoulos, M. Worek, Comput. Phys. Commun. 180, 1941 (2009), 0710.2427

[21] H. Czyz, S. Ivashyn, Comput.Phys.Commun. 182, 1338 (2011), 1009.1881

[22] H. Czyz, E. Nowak-Kubat, Phys. Lett. B634, 493 (2006), hep-ph / 0601169

[23] H. Czyz, E. Nowak-Kubat, Acta Phys. Polon. B36, 3425 (2005), hep-ph/ 0510287

[24] H. Czyz, E. Nowak, Acta Phys. Polon. B34, 5231 (2003), hep-ph/ 0310335 
[25] M. Caffo, H. Czyz, Comput. Phys. Commun. 100, 99 (1997), hep-ph/ 9607357

[26] C.M. Carloni Calame, C. Lunardini, G. Montagna, O. Nicrosini, F. Piccinini, Nucl. Phys. B584, 459 (2000), hep-ph/0003268

[27] C.M. Carloni Calame, Phys. Lett. B520, 16 (2001), hep-ph / 0103117

[28] C.M. Carloni Calame, G. Montagna, O. Nicrosini, F. Piccinini, Nucl. Phys. Proc. Suppl. 131, 48 (2004), hep-ph / 0312014

[29] G. Balossini, C.M. Carloni Calame, G. Montagna, O. Nicrosini, F. Piccinini, Nucl. Phys. B758, 227 (2006), hep-ph/0607181

[30] C. Carloni Calame, H. Czyz, J. Gluza, M. Gunia, G. Montagna et al., JHEP 1107, 126 (2011), 1106.3178

[31] M. Gunia, Acta Phys. Pol. B 42, 2469 (2011)

[32] C. Carloni Calame, H. Czyz, J. Gluza, M. Gunia, G. Montagna et al. (2011), 1112.2851

[33] T. Hahn, M. Perez-Victoria, Comput.Phys.Commun. 118, 153 (1999), hep-ph / 9807565

[34] G.J. van Oldenborgh, Comput. Phys. Commun. 66, 1 (1991)

[35] K. Kajda, T. Sabonis, V. Yundin, Acta Phys. Polon. B40, 3127 (2009), [v40p3127]

[36] G. Passarino, M. Veltman, Nucl. Phys. B160, 151 (1979)

[37] A. Denner, S. Dittmaier, Nucl. Phys. B658, 175 (2003), hep-ph/ 0212259

[38] A. Denner, S. Dittmaier, Nucl. Phys. B734, 62 (2006), hep-ph / 0509141

[39] T. Diakonidis, J. Fleischer, J. Gluza, K. Kajda, T. Riemann et al., Phys.Rev. D80, 036003 (2009), 0812.2134

[40] T. Diakonidis, J. Fleischer, T. Riemann, J.B. Tausk, Phys. Lett. B683, 69 (2010), 0907.2115

[41] J. Fleischer, T. Riemann (2010), arXiv:1009.4436

[42] R.K. Ellis, G. Zanderighi, JHEP 02, 002 (2008), 0712 . 1851

[43] J. Fleischer, T. Riemann, V. Yundin (2011), 1112.0500

[44] V. Yundin, PhD Thesis Humboldt U. in Berlin (2011)

[45] A. Hoefer, J. Gluza, F. Jegerlehner, Eur.Phys.J. C24, 51 (2002), hep-ph/ 0107154 